\title{
AN ABEL-TAUBER THEOREM FOR PARTITIONS
}

\author{
J. L. GELUK
}

ABstract. Suppose $\Lambda=\left\{\lambda_{1}, \lambda_{2}, \ldots\right\}$ is a given set of real numbers such that $0<\lambda_{1}<\lambda_{2}<\ldots$. Let $n(u)=\Sigma_{\lambda_{k}<u} 1$ and $P(u)$ the number of solutions of $n_{1} \lambda_{1}+n_{2} \lambda_{2}+\ldots<u$ in integers $n_{i}>0$. An Abel-Tauber theorem concerning $n(u)$ and $\log P(u)$ is proved for the case where $n(t x) / n(t) \rightarrow 1(t \rightarrow \infty)$ for $x>0$.

Introduction. Suppose $\Lambda=\left\{\lambda_{1}, \lambda_{2}, \ldots\right\}$ is a given set of real numbers such that $0<\lambda_{1}<\lambda_{2}<\ldots$ Let $0=\nu_{0}<\nu_{1}<\ldots$ be the elements of the additive semigroup generated by $\Lambda$. Consider the weighted partition function $p\left(\nu_{m}\right)$ defined by the generating function

$$
\prod_{r=1}^{\infty}\left(1-e^{-\lambda_{r} s}\right)^{-\psi_{r}}=\int_{0}^{\infty} e^{-u s} d P(u):=\hat{P}(s),
$$

where $P(u)=\Sigma_{\nu_{i}<u} p\left(\nu_{i}\right)$ and $\psi_{r}$ is a given sequence with nonnegative terms. We assume that $\lambda_{r}$ and $\psi_{r}$ are such that the product for $\hat{P}(s)$ is (absolutely) convergent for $s>0$.

Letting $n(u)=\Sigma_{\left\{k ; \lambda_{k}<u\right\}} \psi_{k}$ we have the following theorem.

MAIN TheOREM. Let $c$ be a constant, $0<c<\infty$. Under the above assumptions the following two statements are equivalent.

(i) $\lim _{t \rightarrow \infty} n(t)=c$ and $n(t x) / n(t) \rightarrow 1(t \rightarrow \infty)$ for all $x>0$.

(ii) There exists a nondecreasing function $a(t)$ on $(0, \infty)$ with $\lim _{t \rightarrow \infty} a(t)=c$ such that for all $x>0$

$$
\left(\frac{P(t x)}{P(t)}\right)^{1 / a(t)} \rightarrow x \quad(t \rightarrow \infty)
$$

If $c<\infty$ then

$$
\int_{0}^{x} \frac{n(t)}{t} d t-\log P(x) \rightarrow \log \Gamma(1+c) \quad(x \rightarrow \infty) .
$$

If $c=\infty$ then

$$
\log P(y)=\int_{0}^{x} \frac{n(t)}{t} d t+n(x)+o(n(x))
$$

where $x, y \rightarrow \infty$ and $y \sim x n(x) \sim x a(y)$. If $c=\infty$ and the function $n$ satisfies the relation $n(x n(x)) \sim n(x)$ then $n(x) \sim a(x)$ and $\int_{0}^{x}(n(s) / s) d s-\log P(x) \sim n(x)$ $\log n(x)(x \rightarrow \infty)$.

Received by the editors August 8, 1979.

AMS (MOS) subject classifications (1970). Primary 40E05, 26A12.

Key words and phrases. Abel-Tauber theorems, regular variation, partition function.

(C) 1981 American Mathematical Society 0002-9939/81/0000-0362/\$02.25 
In order to prove the Main Theorem we use the Lambert transform $\tilde{n}$ of $n$ which is defined by

$$
\tilde{n}(s)=\int_{0}^{\infty} \frac{s}{e^{u s}-1} n(u) d u,
$$

supposing the integral exists. This transform arises naturally since

$$
\log \hat{P}(s)=-\sum_{r=1}^{\infty} \psi(r) \log \left(1-e^{-\lambda_{r} s}\right)=-\int_{0}^{\infty} \log \left(1-e^{-u s}\right) d n(u)=\tilde{n}(s)
$$

by integration by parts.

We use Abelian and Tauberian theorems connected with the theory of regular variation. Earlier results in this direction were given by Kohlbecker for regularly varying functions and by Parameswaran for slowly varying functions. Our Main Theorem can be seen as a refinement of Parameswaran's work. The main part of the proof is to show the equivalence of the statements (a) $n$ is slowly varying;

(b) $\tilde{n}(1 / x)=\log \hat{P}(1 / x)$ is an element of the class $\Pi$;

(c) $\log (P-1)$ is an element of the class $\Pi$;

(d) $\log P$ is in $\Pi$.

Precise conditions are given in the lemmas.

\section{Results.}

Definition. A function $f: R^{+} \rightarrow R^{+}$is said to be slowly varying if it is measurable and $\lim _{t \rightarrow \infty} f(t x) / f(t)=1$ for $x>0$.

In this paper we use properties of a subclass of the slowly varying functions. This subclass is defined as follows.

Definition. A nondecreasing function $\Pi: R^{+} \rightarrow R^{+}$is said to belong to the class $\Pi$ (notation: $U(\cdot) \in \Pi$ ) if there exists an auxiliary function $a: R^{+} \rightarrow R^{+}$ such that

$$
\lim _{t \rightarrow \infty} \frac{U(t x)-U(t)}{a(t)}=\log x \text { for } x>0 .
$$

The function $a$ is (of course) determined up to asymptotic equivalence. It is possible to show that each function $U(x) \in \Pi$ with $\lim \sup U(x)=\infty$ can be represented as

$$
U(x)=\int_{1}^{x} \frac{L(u)}{u} d u+o(L(x)) \quad(x \rightarrow \infty)
$$

where $L$ is slowly varying. For a proof see [2, Theorem 3].

Conversely each function $U$ of the form (2) satisfies (1). The functions $a, L$ and $U$ are related by $L(x) \sim a(x) \sim(1 / x) \int_{1}^{x} t d U(t)(x \rightarrow \infty)$.

For proofs and properties of regular variation and the class $\Pi$ see [1], [6], [15].

LemMA 1. Suppose $\Sigma_{k<x}(1 / k) n(x / k)=\int_{1}^{x}(L(t) / t) d t+o(L(x)) \quad(x \rightarrow \infty)$, where $L$ is slowly varying, bounded on finite intervals and $\sup _{y<x} L(y)=O(L(x))$ $(x \rightarrow \infty)$. If $x n(x)$ is nondecreasing for $x>1$ then $n(x) \sim L(x)(x \rightarrow \infty)$ and $\int_{1}^{x}[(n(t)-L(t)) / t] d t=-\gamma L(x)+o(L(x))(x \rightarrow \infty)$ where $\gamma$ is Euler's constant. 
Proof. We define $I(x)=\Sigma_{k<x}(1 / k) n(x / k) ; N(x)=\Sigma_{k<x}(\mu(k) / k)(x>0)$; $A(x)=n(x)+\int_{1}^{x}(n(t) / t) d t$ and $a(x)=(1 / x) \int_{1}^{x} A(t) d t$ for $x>1$, where $\mu$ is the Möbius function.

Möbius inversion in the first formula and partial integration in the third gives

$$
n(x)=\sum_{k<x} \frac{\mu(k)}{k} I\left(\frac{x}{k}\right)=\frac{1}{x} \int_{1}^{x} t d A(t) .
$$

Substituting this we get

$$
\begin{aligned}
\int_{1}^{x} \frac{I(x / u) N(u)}{u} d u & =\int_{1}^{x} \frac{I(x / u)}{u} \sum_{k<u} \frac{\mu(k)}{k} d u \\
& =\sum_{k<x} \frac{\mu(k)}{k} \int_{1}^{x / k} I\left(\frac{x}{k u}\right) \frac{d u}{u}=\int_{1}^{x} \frac{1}{u} n\left(\frac{x}{u}\right) d u \\
& =\int_{1}^{x} \frac{n(u)}{u} d u=a(x) .
\end{aligned}
$$

Now for $\lambda>1$ fixed, the function $[I(\lambda x)-I(x)] / L(x)$ is bounded for $x>0$ and tends to $\log \lambda$ for $x \rightarrow \infty$. Substituting the expression for $a(x)$ then gives

$$
\begin{aligned}
\frac{a(\lambda x)-a(x)}{L(x)}= & \int_{1}^{x}\left(\frac{I(\lambda x / u)-I(x / u)}{L(x / u)} \cdot \frac{L(x / u)}{L(x)}\right) \frac{N(u)}{u} d u \\
& +\int_{1}^{\lambda} \frac{I(\lambda / u) N(x u)}{u} d u
\end{aligned}
$$

and the term between brackets in the first integral is bounded for $u \in(1, x)$ by assumption.

As $\int_{1}^{\infty}(N(u) / u) d u$ is absolutely convergent by a classical result of Landau (see [9]), we get by dominated convergence $[a(\lambda x)-a(x)] / L(x) \rightarrow \log \lambda$. Since $x n(x)$ $=\int_{1}^{x} t d A(t)$ is nondecreasing, $A$ is nondecreasing.

This implies that $A \in \Pi$ with auxiliary function $L(x) \sim(1 / x) \int_{1}^{x} t d A(t) \sim n(x)$ by the Main Theorem in the preceding paper (П-Regular Variation). Since $n$ is slowly varying we can apply Theorem 2 in [5] to get

$$
I(x)-\int_{1}^{x} \frac{n(t)}{t} d t=(\gamma+o(1)) n(x) \quad(x \rightarrow \infty) .
$$

Combination with $I(x)=\int_{1}^{x}(L(t) / t) d t+o(L(x))$ then gives the desired result.

REMARK 1. This theorem is an extension of earlier results in [5], [7], [12], [13] and [14]. In the work of Jukes and Segal however, the condition $x \boldsymbol{x}(\boldsymbol{x})$ nondecreasing is only needed for $n(x) \sim L(x)$. Remark that the conditions (see [7])

(i) for some real $r, x g^{\prime}(x)(\log x)^{-r}$ is nonincreasing for $x>x_{0}$, and

(ii) for some real $s, x g^{\prime}(x)(\log x)^{s}$ is nondecreasing for $x>x_{1}$, imply that $x g^{\prime}(x)$ is slowly varying and so $g(x) \in \Pi$ with auxiliary function $x g^{\prime}(x)$.

REMARK 2. If $L(x) \sim M(x)(x \rightarrow \infty)$ where $M(x)$ is nondecreasing, then the condition $\sup _{y<x} L(y)=O(L(x))$ holds.

This is trivial, since $L(y) / L(x) \leqslant \max (A,(1+\varepsilon) M(x)) / L(x)<c$ for all $y<x$, where $L(x) \leqslant(1+\varepsilon) M(x)\left(x \geqslant x_{0}\right), A=\sup _{x<x_{0}} L(x)$ and $c$ is a constant.

This condition is sufficient to prove the Main Theorem. 
For a slowly varying function $\boldsymbol{n}$ the following result gives the relation between $\boldsymbol{n}$ and $\tilde{n}$. For a proof see [5].

LEMMA 2. Suppose $n$ is slowly varying, $n(x) / x$ is integrable on finite subintervals of $R^{+}$. Then $\tilde{n}(1 / x) \in \Pi$ and $\tilde{n}(1 / x)-\int_{0}^{x} n(t) / t d t=o(n(x))(x \rightarrow \infty)$.

A converse statement is given in the next theorem.

LemMA 3. Suppose $n: R^{+} \rightarrow R^{+}$and $n(x) / x$ is integrable on $(0, R)$ for $R>0$, $n(x)$ is nondecreasing for $x>0$ and $\tilde{n}(1 / x)=\int_{1}^{x}(L(u) / u) d u+o(L(x))(x \rightarrow \infty)$, with $L$ slowly varying and asymptotic to a nondecreasing function. Then $n(x) \sim L(x)$ and $\int_{0}^{x}(n(t) / t) d t=\int_{1}^{x}(L(u) / u) d u+o(L(x))(x \rightarrow \infty)$.

Proof. Since $\Sigma_{m<x}(1 / m) n(x / m)$ is nondecreasing, we have $\tilde{n}(1 / x) \in \Pi$ with auxiliary function $L$ iff $\Sigma_{k<x}(1 / k) n(x / k) \in \Pi$ with auxiliary function $L$ (see Corollary 1 in [5]). Morever, $\sum_{k<x}(1 / k) n(x / k)=\tilde{n}(1 / x)-\int_{0}^{1}(n(t) / t) d t+$ $\gamma L(x)+o(L(x))$. Substitution of $\tilde{n}(1 / x)=\int_{1}^{x}(L(u) / u) d u+o(L(x))$ in the expression for $\Sigma_{k<x}(1 / k) n(x / k)$ shows that there exists an $L_{*} \sim L$ such that $\sum_{k<x}(1 / k) n(x / k)=\int_{1}^{x}\left(L_{*}(t) / t\right) d t+o\left(L_{*}(x)\right)(x \rightarrow \infty)$, since $\lim \sup \Sigma_{k \leqslant x}(1 / k) n(x / k)=\infty$. Application of Lemma 1 now gives the desired result.

Proof of The Main Theorem. Suppose $n$ is slowly varying. Application of Lemma 2 now gives $\log \int_{0}^{\infty} e^{-u / x} d P(u)=\tilde{n}(1 / x) \in \Pi$ with auxiliary function $n(x)$. To apply Theorem 1 in [2] we take $P_{0}(y)=-1+P(y)$. Then $P_{0}(0+)=0$ and $\log P \in \Pi$ iff $\log P_{0} \in \Pi$, since $\log P(y)-\log P_{0}(y) \rightarrow 0=o$ (auxiliary function) and the auxiliary function tends to $c>0$. For $c<\infty$ we get $\log P \in \Pi$ with auxiliary function $\sim c$. For $c=\infty$ the result is $\log P(y) \in \Pi$ with auxiliary function $\sim a(y) \sim n(x)$, where $x, y \rightarrow \infty$ are related by $y \sim x n(x) \sim x a(y)$. For the converse statement we replace Lemma 2 by Lemma 3 . All regularity conditions are satisfied since $\boldsymbol{n}$ and $\boldsymbol{P}$ are as mentioned in the introduction. In case $c<\infty$ we combine $\tilde{n}(1 / x)=\int_{0}^{x}(n(t) / t) d t+o(n(x))$ with formula (1.3) in [2]. The case $c=\infty$ is treated similarly with (1.5) in [2]. Now suppose $n(x n(x)) \sim n(x)(x \rightarrow \infty)$. Then $n^{*}(y) \sim 1 / n(y)(y \rightarrow \infty)$ where $n^{*}$ is the conjugate function in the sense of de Bruijn (see [3]). Since $y \sim x n(x) \sim x a(y)$ we get $x \sim y n^{*}(y) \sim y / a(y)$ and so $n(x) \sim a(x)$. This implies that $\log P(t) \in \Pi$ with auxiliary function $n(t)$ satisfying $n(t x) \sim n(t)$ for $t \rightarrow \infty$ uniformly in $x \in[1, n(t)]$ since $n$ is nondecreasing. Application of Theorem 13 in [2] now gives $\log \hat{P}(1 / t)-\log P(t) \sim n(t) \log n(t)$ and the final result follows since $\log \hat{P}(1 / t)=\tilde{n}(1 / t)=\int_{0}^{t}(n(s) / s) d s+o(n(t))(t \rightarrow \infty)$. This finishes the proof.

REMARK 1. The function $n$ satisfies the condition $n(x n(x)) \sim n(x)$ if

$$
\frac{n(2 t)}{n(t)}-1=o(1 / \log n(t)) \text { for } t \rightarrow \infty
$$

REMARK 2. An application to Mahler's partition problem (see [4], [10]) where $\Lambda=\left\{1, r, r^{2}, \ldots\right\}$ and $\psi_{r}=1$ gives

$$
n(u)=\left[\frac{\log u}{\log r}+1\right] \sim \frac{\log u}{\log r} \quad(u \rightarrow \infty) .
$$


Application of the main theorem to this case gives

$$
\log P(u)=\frac{1}{2 \log r} \log ^{2} u-(1+o(1)) \frac{\log u \cdot \log \log u}{\log r}
$$

REMARK 3. If in the case $c=\infty$ and $n(x n(x)) \sim n(x)$ there exists a slowly varying function $L$ such that $L(x) / x$ is convex and $\log P(x)=\int_{0}^{x}(L(t) / t) d t+$ $o(1)$, then we have $\tilde{n}(L(t) / t)+L(t)-\log P(t)-\frac{1}{2} \log 2 \pi L(t) \rightarrow 0(t \rightarrow \infty)$, by Theorem 15 in [2] (in this case $L \sim n$ ). This stronger result is not very useful however, since $\log P$ is expressed in terms of $\tilde{n}$ and not in terms of $n$.

\section{REFERENCES}

1. A. A. Balkema, Monotone transformations and limit laws, Math. Centre Tracts 45, North-Holland, Amsterdam, 1973.

2. A. A. Balkema, J. L. Geluk and L. de Haan, An extension of Karamata's Tauberian theorem and its connection with complementary convex functions, Quart. J. Math. (2) 30 (1979), 385-416.

3. N. G. de Bruyn, Pairs of slowly oscillating functions occurring in asymptotic problems concerning the Laplace transform, Nieuw Arch. Wisk. (3) 7 (1959), 20-26.

4. 210-220.

5. J. L. Geluk, On convolutions with the Möbius function, Proc. Amer. Math. Soc. 77 (1979), 201-210.

.6. L. de Haan, On regular variation and its application to the weak convergence of sample extremes, Math. Centre Tracts 32, North-Holland, Amsterdam, 1970.

7. K. A. Jukes, Tauberian theorems of Landau-Ingham type, J. London Math. Soc. (2) 8 (1974), 570-576.

8. E. E. Kohlbecker, Weak asymptotic properties of partitions, Trans. Amer. Math. Soc. 88 (1958), 346-365.

9. E. G. H. Landau, Handbuch der Lehre von der Verteilung der Primzahlen, Teubner, Leipzig-Berlin, 1909; reprint, Chelsea, New York, 1953.

10. K. Mahler, On a special functional equation, J. London Math. Soc. (1) 15 (1940), 115-123.

11. S. Parameswaran, Partition functions whose logarithms are slowly ascillating, Trans. Amer. Math. Soc. 100 (1961), 217-240.

12. S. L. Segal, On convolutions with the Möbius function, Proc. Amer. Math. Soc. 34 (1972), 365-372.

13. __ A general Tauberian theorem of Landau-Ingham type, Math. Z. 111 (1969), 159-167.

14. Addendum to Jukes' paper on Tauberian theorems of Landau-Ingham type, J. London Math. Soc. (2) 9 (1974), 360-362.

15. E. Seneta, Regularly varying functions, Lecture Notes in Math., vol. 508, Springer-Verlag, 1976.

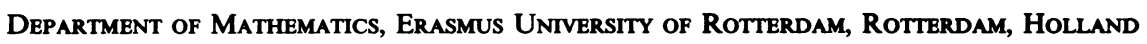

